\title{
Human Mobility from theory to practice: Data, Models and Applications
}

\author{
Filippo Simini \\ Department of Engineering Mathematics, University of \\ Bristol, UK \\ f.simini@bristol.ac.uk \\ Roberto Pellungrini \\ Department of Computer Science, University of Pisa \\ roberto.pellungrini@di.unipi.it
}

\begin{abstract}
The inclusion of tracking technologies in personal devices opened the doors to the analysis of large sets of mobility data like GPS traces and call detail records. This tutorial presents an overview of both modeling principles of human mobility and machine learning models applicable to specific problems. We review the state of the art of five main aspects in human mobility: (1) human mobility data landscape; (2) key measures of individual and collective mobility; (3) generative models at the level of individual, population and mixture of the two; (4) next location prediction algorithms; (5) applications for social good. For each aspect, we show experiments and simulations using the Python library "scikit-mobility" developed by the presenters of the tutorial.
\end{abstract}

\section{CCS CONCEPTS}

- Information systems $\rightarrow$ Data mining; • Computing methodologies $\rightarrow$ Modeling methodologies.

\section{KEYWORDS}

Human Mobility; Artificial Intelligence; Data Science; Generative Models; Predictive Algorithms

ACM Reference Format:

Filippo Simini, Gianni Barlacchi, Roberto Pellungrini, and Luca Pappalardo. 2019. Human Mobility from theory to practice: Data, Models and Applications. In Companion Proceedings of the 2019 World Wide Web Conference (WWW'19 Companion), May 13-17, 2019, San Francisco, CA, USA. ACM, New York, NY, USA, 2 pages. https://doi.org/10.1145/3308560.3320099

\section{TOPIC AND RELEVANCE}

The availability of geo-spatial mobility data (e.g., GPS traces, mobile phone records, social media records) is a trend that will grow in the near future. In particular, this will happen when the shift from traditional vehicles to autonomous, self-driving, vehicles, will change individual and public transportation, transforming our society, the economy and the environment. For this reason, understanding and modeling human mobility is of paramount importance for many

This paper is published under the Creative Commons Attribution 4.0 International (CC-BY 4.0) license. Authors reserve their rights to disseminate the work on their personal and corporate Web sites with the appropriate attribution.

WWW'19 Companion, May 13-17, 2019, San Francisco, CA, USA

(C) 2019 IW3C2 (International World Wide Web Conference Committee), published under Creative Commons CC-BY 4.0 License.

ACM ISBN 978-1-4503-6675-5/19/05.

https://doi.org/10.1145/3308560.3320099

\author{
Gianni Barlacchi \\ Fondazione Bruno Kessler and University of Trento, Italy \\ Povo, Italy \\ gianni.barlacchi@gmail.com \\ Luca Pappalardo \\ ISTI-CNR, Pisa, Italy \\ luca.pappalardo@isti.cnr.it
}

present and future applications such as traffic forecasting, urban planning, estimating migratory flows, and epidemic modeling [12]. In this tutorial we will present a concise and intuitive overview on both the fundamental modeling principles of human mobility and artificial intelligence models applicable to specific mobility-related problems ${ }^{1}$. Starting from the general laws that govern human mobility, we will drive the audience through the main models for human mobility highlighting the parallelism between statistical and deep learning models, presenting the recent advances of the latter that are nowadays representing the state-of-the-art in many human mobility tasks, like next location prediction. To this end, we will review the state of the art of five aspects:

(1) The human mobility data landscape A natural starting point is to describe the nature of empirical data which has been used in mobility research. In this part, we outline the main data sources available for mobility research and the relevant information that can be extracted from them. [3, 4, 6, 17, 35]

(2) Under the microscope: Measuring individual and collective mobility patterns

In this part, we will review some of the fundamental metrics and representations used to characterize human mobility, such as trip distance [8,13], radius of gyration [13, 24, 25], mobility entropy [19, 34], origin-destination matrix [7], mobility motifs [30], and more.

(3) Agents on the move: simulating mobility patterns This part will review the state of the art for generative models at both the individual level (i.e., generation of individual spatio-temporal trajectories) $[2,15,23-25,33]$ and the population level (i.e., generation of mobility flows) [16, 31, 32, 39].

(4) Where's next? AI for human mobility

After a short review of various artificial intelligence and machine learning models for human mobility [21, 22, 36, 38, 43] we will review recent advances based on deep learning, with particular focus on next location prediction $[10,18,40$, 41, 44].

(5) Human mobility for Social Good and future challenges In this part, we will show how developing accurate predictive and generative mobility models can be greatly beneficial for several aspects of social good, from mobility in emergency

\footnotetext{
${ }^{1}$ The online version of the tutorial and all the updated material can be found at https://humanmobility-tutorial.github.io/
} 
scenarios [14] to the prevention of epidemic diffusion [9], nowcast well-being [26] and even the design of more sustainable smart cities [11]. We will discuss about present and future challenges on mobility-related problems such as ridesharing [29], automatic discovery of urban regions [37, 42], prediction of health from human displacements $[1,5]$ and traffic forecasting $[20,28]$. Finally, we discuss privacy issues related to the analysis of human mobility data [27].

\section{ACKNOWLEDGMENTS}

This work is funded by EU project SoBigData RI, grant \#654024.

\section{REFERENCES}

[1] Duygu Balcan, Vittoria Colizza, Bruno Gonçalves, Hao Hu, José J Ramasco, and Alessandro Vespignani. 2009. Multiscale mobility networks and the spatial spreading of infectious diseases. PNAS 106, 51 (2009), 21484-21489.

[2] Hugo Barbosa, Fernando B. de Lima-Neto, Alexandre Evsukoff, and Ronaldo Menezes. 2015. The effect of recency to human mobility. EPf Data Science 4, 1 (2015), 21. https://doi.org/10.1140/epjds/s13688-015-0059-8

[3] Hugo Barbosa and et al. 2018. Human mobility: Models and applications. Physics Reports (2018).

[4] Gianni Barlacchi, Marco De Nadai, Roberto Larcher, Antonio Casella, Cristiana Chitic, Giovanni Torrisi, Fabrizio Antonelli, Alessandro Vespignani, Alex Pentland, and Bruno Lepri. 2015. A multi-source dataset of urban life in the city of Milan and the Province of Trentino. Scientific data 2 (2015), 150055.

[5] Gianni Barlacchi, Christos Perentis, Abhinav Mehrotra, Mirco Musolesi, and Bruno Lepri. 2017. Are you getting sick? Predicting influenza-like symptoms using human mobility behaviors. EPf Data Science 6, 1 (2017), 27.

[6] Vincent D Blondel, Adeline Decuyper, and Gautier Krings. 2015. A survey of results on mobile phone datasets analysis. EPF Data Science 4, 1 (2015), 10.

[7] Patrick Bonnel, Etienne Hombourger, Ana-Maria Olteanu-Raimond, and Zbigniew Smoreda. 2015. Passive Mobile Phone Dataset to Construct Origindestination Matrix: Potentials and Limitations. Transportation Research Procedia 11 (2015), 381 - 398. https://doi.org/10.1016/j.trpro.2015.12.032

[8] D. Brockmann, L. Hufnagel, and T. Geisel. 2006. The scaling laws of human travel Nature 439, 7075 (26 01 2006), 462-465. http://dx.doi.org/10.1038/nature04292

[9] Vittoria Colizza, Alain Barrat, Marc Barthélemy, and Alessandro Vespignani. 2006 The role of the airline transportation network in the prediction and predictability of global epidemics. Proceedings of the National Academy of Sciences 103, 7 (2006), 2015-2020.

[10] Jie Feng, Yong Li, Chao Zhang, Funing Sun, Fanchao Meng, Ang Guo, and Depeng Jin. 2018. DeepMove: Predicting Human Mobility with Attentional Recurrent Networks. (2018).

[11] Michele Ferretti, Gianni Barlacchi, Luca Pappalardo, Lorenzo Lucchini, and Bruno Lepri. 2018. Weak nodes detection in urban transport systems: Planning for resilience in Singapore. arXiv preprint arXiv:1809.07839 (2018).

[12] F. Giannotti, L. Pappalardo, D. Pedreschi, and D. Wang. 2013. A Complexity Science Perspective on Human Mobility. Cambridge University Press, 297âĂŞ314. https://doi.org/10.1017/CBO9781139128926.016

[13] Marta C Gonzalez, Cesar A Hidalgo, and Albert-Laszlo Barabasi. 2008. Understanding individual human mobility patterns. Nature 453, 7196 (2008), 779.

[14] Renhe Jiang, Xuan Song, Zipei Fan, Tianqi Xia, Quanjun Chen, Satoshi Miyazawa, and Ryosuke Shibasaki. 2018. DeepUrbanMomentum: An Online Deep-Learning System for Short-Term Urban Mobility Prediction.. In AAAI.

[15] Shan Jiang, Yingxiang Yang, Siddharth Gupta, Daniele Veneziano, Shounak Athavale, and Marta C. GonzÃąlez. 2016. The TimeGeo modeling framework for urban mobility without travel surveys. PNAS 113, 37 (2016). https://doi.org/10. 1073/pnas.1524261113 arXiv:http://www.pnas.org/content/113/37/E5370.full.pdf

[16] Chaogui Kang, Yu Liu, Diansheng Guo, and Kun Qin. 2015. A Generalized Radiation Model for Human Mobility: Spatial Scale, Searching Direction and Trip Constraint. PLoS ONE 10, 11 (11 2015), 1-11. https://doi.org/10.1371/journal. pone. 0143500

[17] Miao Lin and Wen-Jing Hsu. 2014. Mining GPS data for mobility patterns: A survey. Pervasive and Mobile Computing 12 (2014).

[18] Qiang Liu, Shu Wu, Liang Wang, and Tieniu Tan. 2016. Predicting the Next Location: A Recurrent Model with Spatial and Temporal Contexts.. In AAAI 194-200.

[19] Xin Lu, Erik Wetter, Nita Bharti, Andrew J. Tatem, and Linus Bengtsson. 2013. Approaching the Limit of Predictability in Human Mobility. Scientific Reports 3 (11 10 2013).

[20] Yisheng Lv, Yanjie Duan, Wenwen Kang, Zhengxi Li, and Fei-Yue Wang. 2015. Traffic flow prediction with big data: a deep learning approach. IEEE Transactions on Intelligent Transportation Systems 16, 2 (2015), 865-873.
[21] Wesley Mathew, Ruben Raposo, and Bruno Martins. 2012. Predicting future locations with hidden Markov models. In Procs of the 2012 ACM conference on ubiquitous computing. 911-918.

[22] Anna Monreale, Fabio Pinelli, Roberto Trasarti, and Fosca Giannotti. 2009. Wherenext: a location predictor on trajectory pattern mining. In Procs of the 15th ACM SIGKDD Int Conf on Knowledge discovery and data mining. 637-646.

[23] Luca Pappalardo, Salvatore Rinzivillo, and Filippo Simini. 2016. Human Mobility Modelling: Exploration and Preferential Return Meet the Gravity Model. Procedia Computer Science 83 (2016), 934-939. https://doi.org/10.1016/j.procs.2016.04.188

[24] Luca Pappalardo and Filippo Simini. 2017. Data-driven generation of spatiotemporal routines in human mobility. Data Mining and Knowledge Discovery (2017), 1-43.

[25] Luca Pappalardo, Filippo Simini, Salvatore Rinzivillo, Dino Pedreschi, Fosca Giannotti, and Albert-Laszlo Barabasi. 2015. Returners and explorers dichotomy in human mobility. Nature Comm 6 (2015).

[26] Luca Pappalardo, Maarten Vanhoof, Lorenzo Gabrielli, Zbigniew Smoreda, Dino Pedreschi, and Fosca Giannotti. 2016. An analytical framework to nowcast wellbeing using mobile phone data. International fournal of Data Science and Analytics 2, 1 (01 Dec 2016), 75-92. https://doi.org/10.1007/s41060-016-0013-2

[27] Roberto Pellungrini, Luca Pappalardo, Francesca Pratesi, and Anna Monreale. 2017. A Data Mining Approach to Assess Privacy Risk in Human Mobility Data. ACM Trans. Intell. Syst. Technol. 9, 3, Article 31 (Dec. 2017), 27 pages. https://doi.org/10.1145/3106774

[28] Nicholas G Polson and Vadim O Sokolov. 2017. Deep learning for short-term traffic flow prediction. Transportation Research Part C: Emerging Technologies 79 (2017), 1-17.

[29] Paolo Santi, Giovanni Resta, Michael Szell, Stanislav Sobolevsky, Steven H Strogatz, and Carlo Ratti. 2014. Quantifying the benefits of vehicle pooling with shareability networks. PNAS 111, 37 (2014).

[30] Christian M. Schneider, Vitaly Belik, Thomas Couronné, Zbigniew Smoreda, and Marta C. González. 2013. Unravelling daily human mobility motifs. fournal of The Royal Society Interface 10, 84 (2013). https://doi.org/10.1098/rsif.2013.0246 arXiv:http://rsif.royalsocietypublishing.org/content/10/84/20130246.full.pdf

[31] Filippo Simini, Marta C. Gonzalez, Amos Maritan, and Albert-Laszlo Barabasi. 2012. A universal model for mobility and migration patterns. Nature 484, 7392 (05 04 2012), 96-100. http://dx.doi.org/10.1038/nature10856

[32] Filippo Simini, Amos Maritan, and Zoltán Néda. 2013. Human mobility in a continuum approach. PloS one 8, 3 (2013), e60069.

[33] Chaoming Song, Tal Koren, Pu Wang, and Albert-Laszlo Barabasi. 2010. Modelling the scaling properties of human mobility. Nature Physics 6, 10 (10 2010), 818-823.

[34] Chaoming Song, Zehui Qu, Nicholas Blumm, and Albert-László Barabási. 2010. Limits of Predictability in Human Mobility. Science 327, 5968 (2010), 1018-1021. https://doi.org/10.1126/science.1177170 arXiv:http://science.sciencemag.org/content/327/5968/1018.full.pdf

[35] Laura Spinsanti, Michele Berlingerio, and Luca Pappalardo. 2013. Mobility and Geo-Social Networks. In Mobility Data: Modeling, Management, and Understanding. 315-333.

[36] Dashun Wang, Dino Pedreschi, Chaoming Song, Fosca Giannotti, and AlbertLászló Barabási. 2011. Human mobility, social ties, and link prediction. In Procs of the 17th ACM SIGKDD Int Conf on Knowledge Discovery and Data Mining. 1100-1108. https://doi.org/10.1145/2020408.2020581

[37] Hongjian Wang and Zhenhui Li. 2017. Region Representation Learning via Mobility Flow. In Procs of the 2017 ACM on Conference on Information and Knowledge Management. 237-246.

[38] R. Wu, G. Luo, J. Shao, L. Tian, and C. Peng. 2018. Location prediction on trajectory data: A review. Big Data Mining and Analytics 1, 2 (June 2018), 108-127. https://doi.org/10.26599/BDMA.2018.9020010

[39] Xiao-Yong Yan, Chen Zhao, Ying Fan, Zengru Di, and Wen-Xu Wang. 2014. Universal predictability of mobility patterns in cities. Fournal of The Royal Society Interface 11, 100 (2014), 20140834.

[40] Cheng Yang, Maosong Sun, Wayne Xin Zhao, Zhiyuan Liu, and Edward Y Chang. 2017. A Neural Network Approach to Jointly Modeling Social Networks and Mobile Trajectories. ACM Transactions on Information Systems (TOIS) 35, 4 (2017), 36.

[41] Shuochao Yao, Shaohan Hu, Yiran Zhao, Aston Zhang, and Tarek Abdelzaher. 2017. Deepsense: A unified deep learning framework for time-series mobile sensing data processing. In Procs of the 26th Int Conf on World Wide Web. 351360.

[42] Nicholas Jing Yuan, Yu Zheng, and Xing Xie. 2018. Discovering Functional Zones in a City Using Human Movements and Points of Interest. In Spatial Analysis and Location Modeling in Urban and Regional Systems. Springer, 33-62.

[43] Chao Zhang, Keyang Zhang, Quan Yuan, Luming Zhang, Tim Hanratty, and Jiawei Han. 2016. Gmove: Group-level mobility modeling using geo-tagged social media. In Procs of the 22nd ACM SIGKDD Int Conf on Knowledge Discovery and Data Mining. 1305-1314.

[44] Junbo Zhang, Yu Zheng, and Dekang Qi. 2017. Deep Spatio-Temporal Residual Networks for Citywide Crowd Flows Prediction.. In AAAI. 1655-1661. 